\title{
Posterior chamber intraocular lens implantation-a new forceps to simplify capsular bag fixation
}

\author{
RICHARD BATES \\ From Whipps Cross Hospital, London E11
}

SUMMARY The case for capsular bag fixation of the Sinsky-Kratz type of posterior chamber intraocular implant lens is presented, and the various methods in use to position implant lenses are outlined. The new forceps are described and the rationale for their adoption and use is given in an illustrated, step-by-step fashion. Their additional use in anterior chamber intraocular lens implantation is mentioned. There is a brief summary of the author's experience with this instrument.

This paper presents an instrument based on a new design concept which simplifies the insertion of posterior chamber intraocular implant lenses. It has also proved useful in positioning anterior chamber lenses of certain designs.

Extracapsular cataract extraction (ECCE) is increasingly accepted as the best method of management of cataract, particularly in patients for whom intraocular lens (IOL) implantation is planned. So safe is the procedure of extracapsular cataract extraction and posterior chamber intraocular lens implant (ECCE and PCIOL), in terms of sparing of corneal endothelial cells and the absence of surgical complications $^{13}$ that the minimum age for lens implantation is falling, and some surgeons are prepared to undertake this procedure for carefully selected patients of 30 years or younger.

The three principal aims of ECCE and PCIOL technique are: (1) to minimise intraocular instrumentation; (2) to limit corneal bending; (3) to implant in such a way as to minimise deleterious interaction between lens and ocular tissues (some authorities would add that this is best achieved by intracapsular IOL fixation-bag fixation. Bag fixation is the most physiological position for the pseudophacos and its advantages are increasingly well documented. ${ }^{+7}$

\section{Description of instrument}

The forceps illustrated are for right-handed surgeons; a left-hander would require a mirror image

Correspondence to Mr R A Bates, Eyc Unit, Whipps Cross Hospital, London E11 1NR. instrument. Their design embodies unique features. A small soft action forceps body of surgical stainless steel (Fig. 1A) resistant to torsion was chosen. The jaws have two bends, the most proximal such that the jaws face forwards with the handle held in the palm between forefinger and thumb. At this bend there is an offset so that the action of the tips is a slide rather than a pure apposition. The tips of the forceps (Fig. 1B) are hooked downwards at $90^{\circ}$. The upper, anterior jaw has a shallow notch cut horizontally close to the tip to enable a $0.4 \mathrm{~mm}$ loop (haptic) to be grasped. The lower, posterior jaw is polished and profiled to act as a ramp to deflect the loop posteriorly as well as superiorly as it is released (patient in the anatomical position). The posterior jaw's hooked shape also acts as an iris and capsule retractor if necessary.

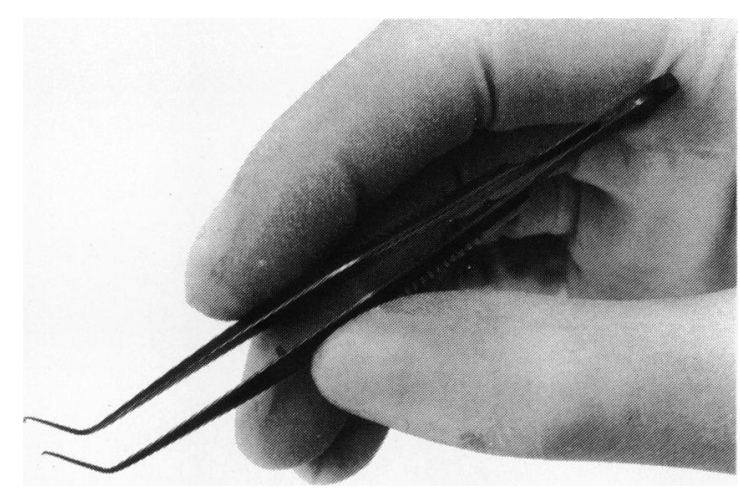

Fig. 1A The new forceps. 


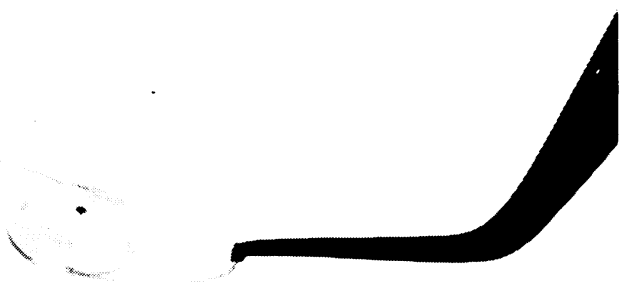

Fig. 1B Detail of the tips grasping an IOL loop.

The chief advantage of these forceps over current designs $^{\text {By }}$ is that bag placement of the PCIOL is possible with a one-handed technique ${ }^{1011}$ and dialling is avoided.

\section{Use of instrument}

A routine extracapsular cataract extraction is performed. I prefer a corneal section placed just anterior to the limbal vascular arcades, but surgeons using a corneoscleral approach will find the forceps similarly useful. The anterior chamber is deepened with viscoelastic fluid to open as far as possible the fornices of the capsular bag. The IOL is presented to the section held either conventionally in Clayman's forceps (Fig. 2) or the author's forceps turned upside down, and the lower loop is passed across the anterior chamber, steeply angled so that the apex of the loop glides over the posterior capsule (with care if a $1^{\circ}$ posterior capsulotomy has been fashioned) until it rests in the inferior capsular fornix. The lens is released before the forceps enter the eye, and the apex of the upper loop is grasped with the implant forceps, the loop being engaged in the groove of the

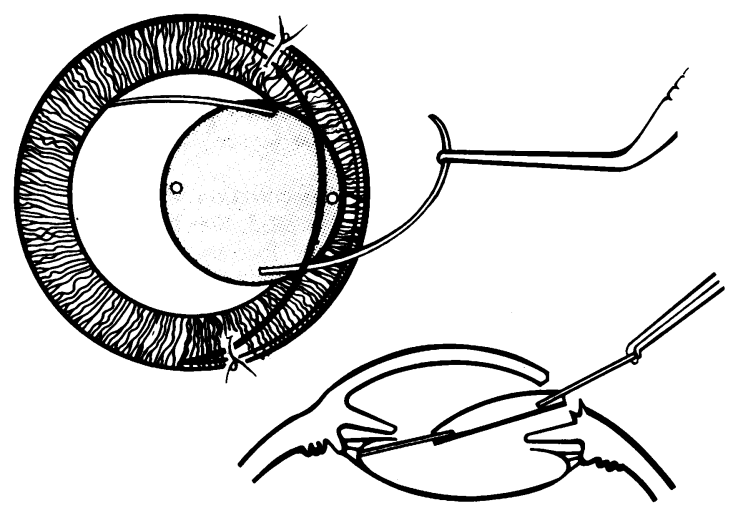

Fig. 3. Method of grasping the superior loop.

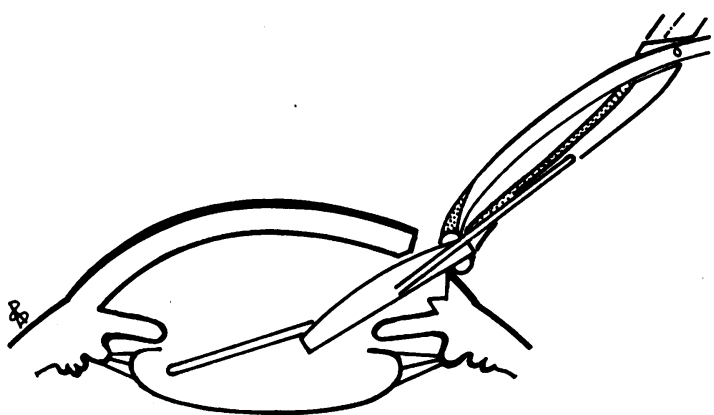

Fig. 2 The inferior loop of the IOL positioned in the bag with Clayman's forceps.

anterior jaw (Fig. 3). By advancing the forceps into the anterior chamber both loops are compressed until the optic lies behind the pupillary aperture and the apex of the upper loop will pass behind the iris (Fig. 4). Note that by holding the upper loop rather than the optic for this part of the manoeuvre the elasticity of both loops comes into play, and the risk of zonular dehiscence or capsular rupture is minimised.

The surgeon rotates the forceps between finger and thumb, steepening the angle of the jaws and using the point of entry into the eye as the centre of rotation, taking care not to open the section or bend the cornea. This allows the superior loop to slide away from the jaw of the forceps and retracts the iris and anterior capsular remnant if necessary (Fig. 5). When the loop is released the posterior jaw acts as a chute diverting it into the upper bag fornix. Fig. 6 shows the correct position of the lens following removal of the forceps. A bag fixated IOL will require no formal dialling to centre it. Gentle nudging with the tip of the dialling hook to ensure the posterior capsule is well stretched and not puckered is sufficient.

When the anterior chamber is reformed, the bag fixated IOL will be seen to lie very posteriorly,

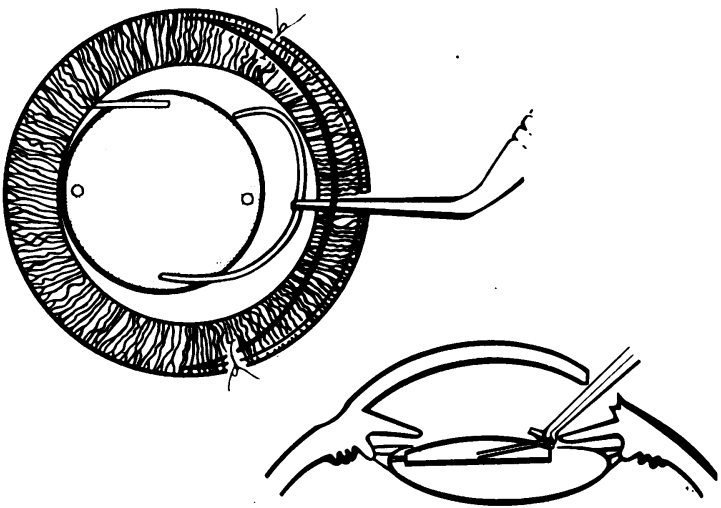

Fig. 4 Both loops compressed. 


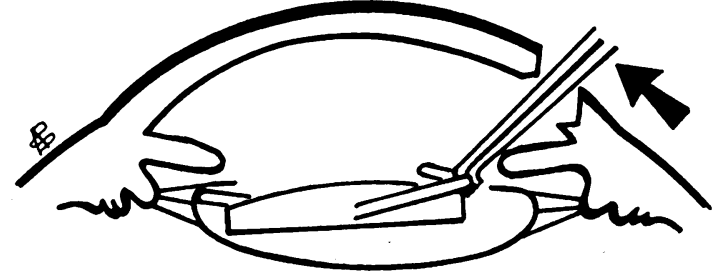

Fig. 5 Steeping the angle of the jaws before releasing the loop.

allowing a clear gap between iris and optic in most eyes.

\section{Experience with instrument}

The author's experience with this instrument consists of a series of 150 posterior chamber Sinsky-Kratz style implant lenses of various manufacture, the last 73 of which were intended to be bag fixated. Three implants were of the Arnott AR4 type, and one was a closed-loop Sheets design. Seven patients had $1^{\circ}$ capsulotomy immediately prior to implantation for central capsular fibrosis. Two patients' implants were inserted as $2^{\circ}$ procedures and bag fixation was naturally not possible. Of the 73 lenses intended to be bag fixated, 68 appeared on the table to be successfully placed, and one further patient subsequently appeared by three-mirror Goldmann lens examination through fully dilated pupil to have a sulcus fixated lens.

One highly myopic patient, having had a sulcus fixated (+6.0D) IOL, failed to achieve IOL fixation, and at six weeks the IOL was freely mobile in the retropupillary anterior segment. This was removed without difficulty and without affecting visual acuity; the eye had macular myopic degeneration.

The position of all lenses was confirmed within the first six weeks following surgery, by which time loops of the bag fixated IOLs were firmly encased within the adherent capsular leaves. All bag fixated IOLs appeared well centred; no dialling holes or edges of optics were visible through undilated pupils.

The versatility of this instrument allows its use in the placement of the upper loop of semiflexible or semirigid open loop anterior chamber IOLs such as the Novaflex, Optiflex, or Multiflex type. The author has performed only a small number of anterior chamber implants, but sufficient to confirm the instrument's usefulness in this type of surgery in placing the superior loop without excessive pressure on the inferior angle structures.

In conclusion this instrument has been designed specifically to meet the needs of superior loop placement of the Sinsky-Kratz posterior chamber IOL with minimal intraocular manipulation, and it

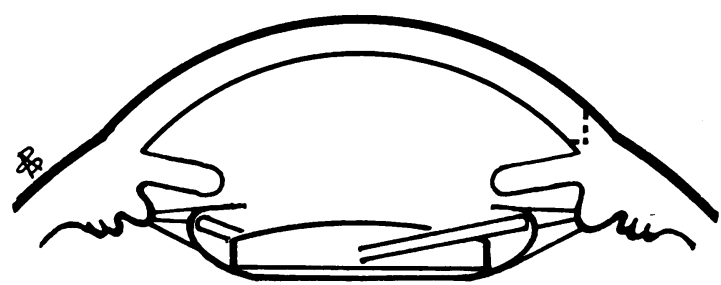

Fig. 6 Correct positioning of a bag fixated IOL.

facilitates capsular bag fixation, which can be reliably achieved with a one-handed technique. No torsional force is applied to the lens as with conventional technique, and the danger of inadvertant IOLendothelial touch is avoided. Dialling is unnecessary, and iris trauma is minimised.

The longer follow-up of the series of patients in whom bag fixation of the IOL was attempted will be the subject of a further report.

I acknowledge with gratitude the assistance of the consultant staff of Whipps Cross Hospital Eye Unit: Mr J S Conway, Miss M T Challis, and Mr B Beveridge, whose patients comprise my series; Mr A D McG Steele for his constructive criticism; Miss Karen Johnston and Mr Max Brown of the Medical Illustration Department, Moorfields Eye Hospital.

The implant forceps are commercially available from Osborne and Simmonds, London EC1R OAT. My thanks to Mr H Simmonds for his work on this instrument.

\section{References}

1 Kraff MC, Sanders DR, Liebermann HL. Monitoring for continuing endothelial cell loss with cataract extraction and intraocular lens implantation. Ophthalmology (Rochester) 1982; 89: $30-4$.

2 Oxford Cataract Treatment and Evaluation Team. Complications of cataract surgery: results of a randomised controlled clinical trial. Trans Ophthalmol Soc UK in press.

3 Percival SBP. Long term complications from extracapsular cataract surgery. Trans Ophthalmol Soc UK 1985; 104: 915-8.

4 Sheets JH. Capsular fixation of posterior chamber intraocular lenses. Arnott EJ, eds. In Intraocular lens implantation. St Louis: Mosby, 1984.

5 Miyike K, Asakura M, Kobayashi H. Effect of intraocular lens fixation on the blood-aqueous barrier. Am J Ophthalmol 1984; 98: 451-5.

6 Samples JR, Van Buskirk M. Pigmentary glaucoma associated with posterior chamber intraocular lenses. Am J Ophthalmol 1985; 100: 385-8.

7 Green WR, McDonnell PJ. Opacification of the posterior capsule. Trans Ophthalmol Soc UK 1985; 104: 727-39.

8 Clayman HM. Lens implant forceps. Ophthalmology (Rochester) 1977; 83: 853-4.

9 Simcoc CW. Lens insertion forceps. J Am Intraocul Implant Soc 1980; 6: 60 .

10 Clayman HM. Technique for the insertion of the superior loop of the Shearing-style posterior chamber lens. J Am Intraocul Implant Soc 1980; 6: 383-4

11 Sinsky RM. Posterior chamber lens modification. J Am Intraocul Implant Soc 1981; 7: 260-1.

Accepted for publication 24 January 1986. 